\title{
Collaborative effects of wavefront shaping and optical clearing agent in optical coherence tomography
}

Hyeonseung $\mathrm{Yu}$

Peter Lee

YoungJu Jo

KyeoReh Lee

Valery V. Tuchin

Yong Jeong

YongKeun Park 


\title{
Collaborative effects of wavefront shaping and optical clearing agent in optical coherence tomography
}

\author{
Hyeonseung Yu, a,b Peter Lee, ${ }^{\mathrm{b}, c}$ YoungJu Jo, ${ }^{\mathrm{a}, \mathrm{b}}$ KyeoReh Lee, ${ }^{\mathrm{a}, \mathrm{b}}$ Valery V. Tuchin, ${ }^{\text {de, }, \mathrm{f}}$ Yong Jeong, ${ }^{\mathrm{b}, \mathrm{c}}$ and \\ YongKeun Park ${ }^{a, b, *}$ \\ ${ }^{a}$ Korea Advanced Institute of Science and Technology, Department of Physics, 291 Daehak-ro, Yuseong-gu, Daejeon 34141, Republic of Korea \\ ${ }^{b}$ KAIST Institute of Health Science and Technology, 291 Daehak-ro, Yuseong-gu, Daejeon 34141, Republic of Korea \\ 'Korea Advanced Institute of Science and Technology, Department of Bio and Brain Engineering, 291 Daehak-ro, Yuseong-gu, Daejeon 34141, \\ Republic of Korea \\ 'Saratov National Research State University, Research-Education Institute of Optics and Biophotonics, 83, Astrakhanskaya Street, \\ Saratov 410012, Russia \\ eInstitute of Precision Mechanics and Control of Russian Academy of Sciences, Laboratory of Laser Diagnostics of Technical and Living Systems, \\ 24, Rabochaya Street, Saratov 410028, Russia \\ fNational Research Tomsk State University, Laboratory of Biophotonics, 36, Lenin's Avenue, Tomsk 634050, Russia
}

\begin{abstract}
We demonstrate that simultaneous application of optical clearing agents (OCAs) and complex wavefront shaping in optical coherence tomography (OCT) can provide significant enhancement of penetration depth and imaging quality. OCA reduces optical inhomogeneity of a highly scattering sample, and the wavefront shaping of illumination light controls multiple scattering, resulting in an enhancement of the penetration depth and signal-to-noise ratio. A tissue phantom study shows that concurrent applications of OCA and wavefront shaping successfully operate in OCT imaging. The penetration depth enhancement is further demonstrated for ex vivo mouse ears, revealing hidden structures inaccessible with conventional OCT imaging. ๑ 2016 Society of Photo-Optical Instrumentation Engineers (SPIE) [DOI: 10.1117/1.JBO.21.12.121510]
\end{abstract}

Keywords: optical coherence tomography; imaging through turbid media; active or adaptive optics.

Paper 160311SSRR received May 17, 2016; accepted for publication Oct. 5, 2016; published online Oct. 28, 2016.

\section{Introduction}

Developing a deep-tissue imaging tool with good optical resolution is one of the important goals of biophotonics because medical diagnosis usually requires the imaging of fine details of whole tissue structures. Optical coherence tomography (OCT) is an extensively developed imaging tool for biological tissues. Based on low-coherence interferometry, OCT provides micrometer scale resolution and enables noninvasive high-speed imaging. ${ }^{1}$ OCT is widely used in ophthalmology because it can fully access retina, choroid, and sclera layers of human eyes in vivo. ${ }^{2,3}$ OCT has a higher penetration depth of 1 to $2 \mathrm{~mm}$ compared to other optical imaging modalities such as multiphoton microscopy and confocal microscopy. ${ }^{4}$ Photoacoustic microscopy also offers deep tissue imaging capability, but optical absorption contrast is used instead of elastic backscattering signals. ${ }^{5}$ Despite its advantages, OCT cannot provide penetration depth on centimeter scales as is possible with ultrasound imaging.

The limitation of the penetration depth of OCT originates from multiple light scattering caused by inhomogeneous refractive index distributions in biological tissues. The strategy of OCT imaging is to deliver light deep into a target region and measure backscattered light. However, light is severely scattered during propagation inside a biological tissue. Biological tissues are composed of various cellular components, each having different chemical compositions and thus different refractive indices. For example, cellular organelles, cytoplasm, and collagen fibers have refractive indices higher than that of interstitial fluid. More quantitatively, optical waves have a reduced scattering coefficient of
$1 \mathrm{~mm}^{-1}$ in most biological tissues. ${ }^{6}$ This implies that after propagation of $1 \mathrm{~mm}$, the light experiences a few scattering events (on average ten for the scattering anisotropy factor typical for tissues, $g=0.9$ ), after which the light completely loses its original propagation direction. To minimize the effect of multiple scattering events, OCT uses coherence gating to selectively collect scattering signals with the same optical path lengths. However, the amount of single-scattered light exponentially decays as the depth increases, and multiple scattering becomes dominant beyond a few mean free paths. ${ }^{7}$ Thus, it is extremely challenging to image scatterers at depths greater than $\sim 1 \mathrm{~mm}$; thus, penetration depth is limited to a few millimeters.

One method of visualizing deep inside tissues in OCT systems is to utilize optical clearing agents (OCAs) to compensate for optical inhomogeneity. ${ }^{8,9}$ The primary role of OCAs is to reduce refractive index mismatches among tissue structures and thus to reduce light scattering in the tissues. ${ }^{10-12}$ Due to the complexity of tissue structures, the reduction of the scattering cannot be explained merely by the refractive indices of the OCAs. ${ }^{13,14}$ In fact, various mechanisms are involved, such as the dissociation of collagen, ${ }^{15,16}$ the ability to disrupt the hydration shell and the water-mediated hydrogen bonds of collagen molecules, ${ }^{17}$ and the dehydration process. ${ }^{10,18-20}$ In addition to penetration depth enhancement for tissue imaging, ${ }^{21-23}$ OCAs also enable optical blood clearing ${ }^{24}$ and monitoring of molecular diffusion $^{25-30}$ using OCT systems.

Although OCA approaches use the scattering properties of tissue samples for the enhancement of the penetration depth, it has been recently demonstrated that controlling the illumination 
(a)

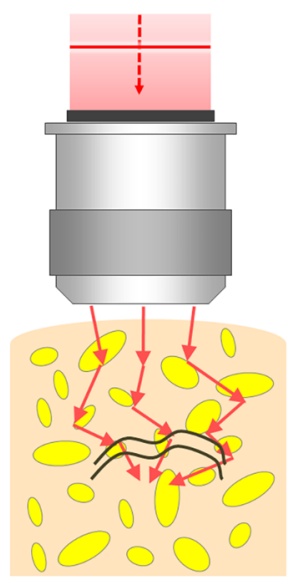

(b)

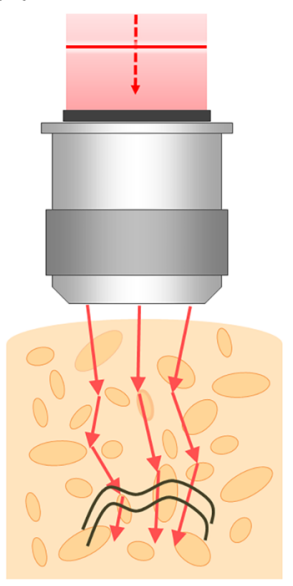

(c)

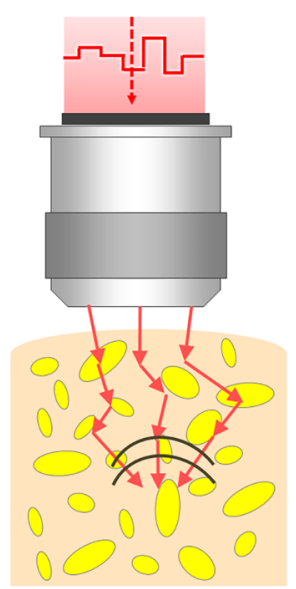

(d)

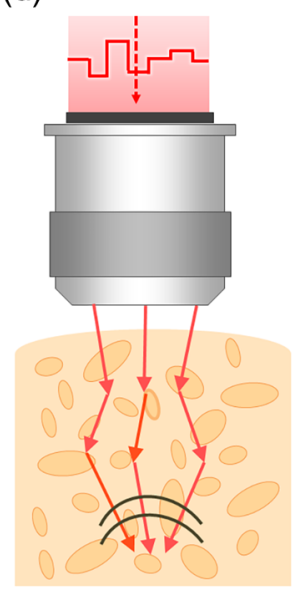

Fig. 1 Principle of OCAs and wavefront shaping in an OCT system. (a) Conventional OCT imaging. (b) After the application of OCAs, the overall scattering of the tissue is reduced. (c) Wavefront shaping in OCT. The optimal incident wavefront generates a tight focus inside the tissue. (d) Combined effect of OCAs and wavefront shaping. Maximal penetration depth can be achieved with reduced scattering of the sample and optimal incident light field.

light in optical imaging can offer a high possibility of overcoming multiple scattering without affecting the sample. Although adaptive optics approaches were exploited to correct aberrations, the multiple light scattering requires more degrees of freedom. ${ }^{31,32}$ The formation of optical focus can be interpreted as constructive interference of scattered light waves. Multiple light scattering scrambles phase information of an impinging beam, thus the desired constructive interference, or optical focus, cannot be achieved deep in a tissue. However, these perturbations can be adjusted by controlling the complex field of the incident light. ${ }^{33,34}$ This idea was first demonstrated by showing that clear optical focus can be formed through highly scattering media; ${ }^{35}$ later, this wavefront shaping approach was extended to optical systems that are based on OCT principles. The focusing of light into deep tissue was demonstrated using optical coherence microscopy ${ }^{36}$ and penetration depth enhancement was studied in a spectral domain OCT (SD-OCT) system, ${ }^{37-39}$ the so-called wavefront shaping OCT (WS-OCT). The measurement of a time-resolved reflection matrix based on the principles of timedomain OCT enabled selective focusing inside tissues ${ }^{40}$ and diffraction-limited imaging. ${ }^{41,42}$ In a related field, wavefront shaping approaches have also been exploited for deep-tissue imaging with multiphoton microscopy. ${ }^{43,44}$ One of the advantages of using wavefront shaping in OCT is that the light control can also compensate system aberrations or the degradation in resolutions at out-of-focus planes. ${ }^{45}$

In this work, we demonstrate that simultaneous applications of wavefront shaping and OCAs can provide collaborative effects for enhancing the penetration depth using WS-OCT. Even though both the wavefront shaping and OCAs enhance the penetration depth, the collaborative effect is not straightforward due to the loss of the scattering contrast by OCAs. Because the wavefront shaping technique utilizes the backscattered signals for optimization, the reduced scattering by OCAs should be still strong enough so that the wavefront shaping works. A tissue-mimicking phantom and an ex vivo mouse ear tissue were visualized with the application of $70 \%$ glycerol solution and wavefront shaping via a digital micromirror device (DMD). Benefitting from balanced applications of the OCAs and the wavefront shaping, the collaborative effects of both methods offer unprecedented enhancement of the penetration depth compared to that possible with conventional SD-OCT imaging.

\section{Principles}

The principles of OCA and wavefront shaping in OCT are shown in Fig. 1. In a conventional OCT system, an uncontrolled beam is incident on a sample and backscattered signals from a scatterer are used as an image contrast. The desired situation is one in which a tight focus is formed at a target axial depth and signals are strongly reflected from the focus, providing vivid images of tissue structures. However, due to optical inhomogeneity inside the tissue, the beam propagation paths that are supposed to be focused on the target spot experience spatially varying perturbations.

This situation leads to severe degradation of the optical focus, as shown in Fig. 1(a). There are two different approaches that can alleviate this degradation of the focus: the application of OCA, as shown in Fig. 1(b), and wavefront shaping, as shown in Fig. 1(c). In Fig. 1(b), OCA can be seen to reduce the inhomogeneity of the tissue, and the multiple scattering becomes less severe. The penetration depth can be extended at the expense of the tissue composition change. In contrast to the OCA approach, which directly changes the sample, the wavefront shaping method controls light propagation in tissues to maximize the light delivery to scatters and the reflection from the scatters, as shown in Fig. 1(c). Because the wave-propagation in tissues is a deterministic process, we can find an optimal wavefront that can selectively focus at the target depth inside the tissue. While the OCA directly suppresses multiple light scattering by manipulating the tissue, wavefront shaping controls the light paths without affecting the tissue structure. Therefore, these two approaches can work concurrently, and the combination of both methods provides collaborative effects to control multiple light scattering. This best situation of the combined approach is shown in Fig. 1(d). For all the above cases, the enhancement of the penetration depth can be maximized when the OCA technique and the wavefront shaping approaches are simultaneously applied. 


\section{Experimental Setup}

A schematic of the experimental setup is shown in Fig. 2. The system was adapted from an SD-OCT system by incorporating high-speed wavefront modulation. ${ }^{37}$ In a conventional system, a collimated beam from an illumination source directly passes through a beam splitter and the divided beams arrive incident on a sample and a reference mirror, respectively. For the WS-OCT, a wavefront shaping unit consisting of a DMD and a dispersion compensating grating $(\mathrm{G} 1)$ is additionally installed, as indicated by the gray dashed rectangular area. After the wavefront shaping unit, OCT signals are acquired and processed based on SD-OCT principles. Detailed theories on SD-OCT systems can be found in the literature. ${ }^{45}$

In the WS-OCT system, a broadband superluminescent diode (SLD-52, Superlum Diodes, Ltd., Ireland) with a center wavelength of $1025 \mathrm{~nm}$ (full width at half maximum $=100 \mathrm{~nm}$, $9.7 \mathrm{~mW}$ ) is used as the light source. The beam propagates in free space after the collimator and impinges on the DMD (0.7" XGA, $1024 \times 768$, Texas Instruments). The DMD is a two-dimensional (2-D) array of 800k micromirrors; each mirror directs the incident beam at 12 or $-12 \mathrm{deg}$. The light redirected from the DMD with the angle of $12 \mathrm{deg}$ propagates along the optical axis; the light redirected with the angle of -12 deg is blocked out. Thus, the control of individual micromirrors provides spatially structured incident beams. Due to the pixelated periodic structure, the DMD essentially works as a grating. For broadband illumination, the dispersion is produced and is therefore compensated for by the grating (300 lines/mm, GR250310, Thorlabs). The modulated field pattern is delivered to the back pupil of an objective lens (LSM-03BB, Thorlabs) through two 4- $f$ systems. The modulated incident beam is divided into a sample beam and a reference beam at the beam splitter, and then the reflected light from the sample and the mirror are collected by a single-mode fiber. At the output facet of the collection fiber, interference signals of the sample and reference beams are analyzed by a spectrometer system consisting of a grating (1200 lines/mm, Wasatch Photonics) and a line CCD (SU1024, 1024 lines, 92-kHz frame rate, Sensors Unlimited Inc.). A 2-D image is acquired by steering the sample beam with one mirror of a two-axis galvo mirror $(6210 \mathrm{H}$,
Cambridge Technology), whereas the other mirror is unused. Because the DMD plane is conjugated with the scanning mirror plane, the amplitude modulation at the DMD plane is converted to full-field modulation at the sample plane. In the setup, the reference beam is also modulated by the DMD to preserve the simplicity of the setup. It is possible in principle to separate the reference beam and sample beam, but matching the optical path lengths between the separated sample and reference beams requires a more complicated setup. We also considered that simultaneous modulation of both arms does not result in the difference in the acquired signal, because the interference signal is a function of the temporal frequency, whereas the DMD modulates the spatial profile of the incident beam. The spatial modulation of the reference beam only changes the coupling efficiency to the single-mode fiber, which is calibrated in the signal processing step.

The goal of wavefront shaping optimization is to maximize the reflected signal from the desired depth. Because tissue compositions are entirely unpredictable, characterization of the optical responses of the tissues is required to find the optimal wavefront. Furthermore, tissue structures vary spatially. Thus, the optimization is sequentially processed for each A-scan. In the optimization of a single A-scan, the detailed procedures are divided into three stages: (1) recording of OCT signals with Hadamard pattern projections to characterize the tissue response, (2) calculation of optimal DMD patterns, and (3) recording of enhanced signals for projections of optimal DMD patterns.

At first, 7500 2-D Hadamard patterns are sequentially projected on the DMD and A-scan profiles are recorded for each pattern. Hadamard patterns are mutually independent, and this property minimizes the redundancy of the image acquisitions. Second, we select 200 depth positions as regions of interest (ROI) in the A-scan and individually calculate the optimal pattern at each depth. Here, the axial resolution is $2.9 \mu \mathrm{m}$ and 200 pixels ROI corresponds to a $580-\mu \mathrm{m}$ depth range. In the calculation of the optimal DMD pattern at a specific depth, we selectively choose 500 Hadamard patterns out of the 7500 initial patterns that give the largest signal at the given depth. Because the coherent control of light scattering exploits the linear relation between the input and reflected light fields, raw signals are manipulated in a linear scale. After that, we coherently sum

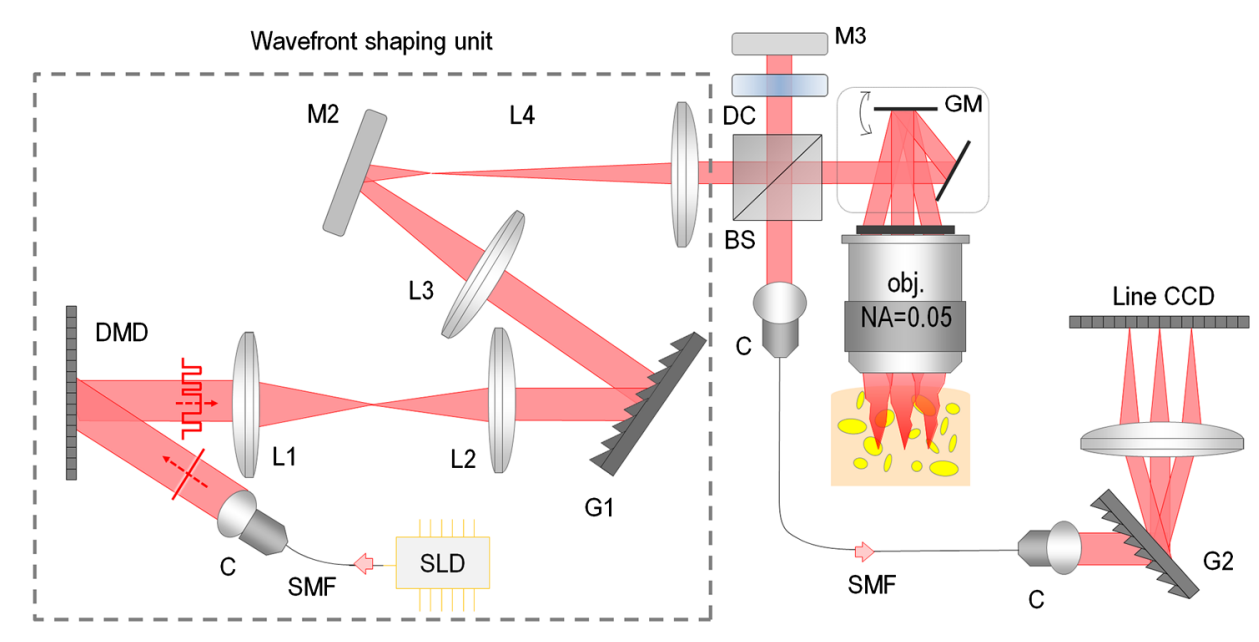

Fig. 2 Experimental setup. Gray dashed rectangular area: wavefront shaping unit: SLD, super-luminescent diode, DMD, digital micromirror device, G1, dispersion compensating grating; BS, beam splitter, DC, dispersion compensator, GM, galvo mirror, G2, spectral OCT grating; C, collimators, L, lenses, $\mathrm{M}$, mirrors, SMF, single-mode fiber. The intensity modulation after the DMD is not to scale. 
the selected 500 patterns to produce a single-optimal pattern. Consequently, we have a total of 200 optimal patterns that individually generate the optimal signal at each depth of the ROI. Third, the calculated 200 optimal patterns are projected sequentially on the DMD, and the enhanced OCT signals are recorded. The recorded $200 \mathrm{~A}$-scan signals contain the enhanced signals at different depth positions, so they are compounded into a single A-scan image in which all pixels in the ROI have the enhanced signal. The acquisition time of a single-optimized A-scan image is $15 \mathrm{~s}$, and $25 \mathrm{~A}$-scans are acquired to obtain a 2-D image.

To demonstrate the coherent control effect of the wavefront shaping, we also acquired the images using the spatial compounding method, which provides a way of suppressing coherent speckle noises by incoherently averaging several OCT signals from spatially or temporally varying illuminations. ${ }^{46-49}$ In our case, 25 random binary DMD patterns were illuminated on the sample, and the 25 acquired OCT signals were incoherently summed to reduce the noise. To properly compare the spatial compounding method and the wavefront shaping, the size of the macropixels in the DMD and the camera acquisition settings are set to be the same for both cases.

\section{Tissue Phantom Study}

To validate the idea of the combined effect of OCA and wavefront shaping for the penetration depth enhancement, we first conducted experiments on a tissue phantom fabricated with $20 \%$ intralipid and agar. ${ }^{50}$ Among various tissue phantom models, ${ }^{51}$ the used phantom has several advantages: (1) the good permeability of agarose enables the efficient diffusion of OCAs and (2) the scattering coefficient of the tissue phantom is easily tuned by changing the weight/volume fraction of the intralipid

\subsection{Sample Preparation}

For the fabrication of the tissue phantom, agarose solution is first prepared by dissolving $1 \mathrm{~g}$ of agarose powder (A5304$100 \mathrm{G}$, Sigma-Aldrich) in $100 \mathrm{ml}$ of distilled water at $95^{\circ} \mathrm{C}$. Then $20 \%$ intralipid (Fresenius Kabi AB, Sweden) and agarose solution are homogenously mixed at a $2 \%$ weight/volume fraction at $75^{\circ} \mathrm{C}$. After the temperature of the mixture reverts to room temperature, the mixture spontaneously becomes a solid-state. The reduced scattering coefficient of the tissue phantom is measured and found to be $1.21 \pm 0.13 \mathrm{~mm}^{-1}$; this value is obtained by integrating the sphere measurement and inverseadding doubling methods. ${ }^{52}$ Most biological tissues have reduced scattering coefficients of $\sim 1 \mathrm{~mm}^{-1}$, which is comparable to the value of the fabricated tissue phantom. ${ }^{6}$ We note that the scattering properties of the use tissue phantom are not identical to those of biological tissues. The anisotropy factor of intralipid is 0.52 at $1064 \mathrm{~nm}$ wavelength. ${ }^{53}$ In general, biological tissues have anisotropy factors of 0.8 to $1.0 .{ }^{6}$ Glycerol, a $70 \%$ solution in water, was used as an OCA. The refractive indices of intralipid and agarose are 1.357 (Ref. 53) and 1.3348 (Ref. 54) at $1064 \mathrm{~nm}$, respectively. From the law of Gladstone and Dale, the mean refractive index of the tissue phantom is estimated as 1.3392. The scattering particles in intralipid are soybean oil, and its refractive index is $1.460 .{ }^{12}$ When the glycerol with the RI of 1.463 is applied to the tissue, it is expected to match the refractive indices of the background and soybean oil by increasing the refractive index of the background.

The target imaging sample has a layered structure: layer $I$ is the tissue phantom, layer III is a translucent Petri dish, and layer
II is the boundary between layer I and layer III, as shown in Fig. 3(a).

\subsection{Experimental Results}

To demonstrate the effect of the OCA and the wavefront shaping, we compared wavefront shaping and conventional images of the tissue phantom before applying OCA and after applying OCA. A photograph of the sample before the application of the OCA is shown in Fig. 3(b). The tissue phantom layer is fabricated to have a slope so that the left part is thinner than the right part. Due to the small thickness, the left part looks more grayish than the right part, but there is still a clear difference in the color between the left part of the sample and the Petri dish. When the OCA was applied to the left thinnest part, reduction in scattering made the layer almost transparent, and the Petri dish below became visible, as shown in Fig. 3(c). This clearing effect is more clearly visible in the insets with the magnified images of the edge. Before applying the glycerol OCA, the wavefront shaping and uncontrolled illumination results are acquired as depicted in Figs. 3(d)-3(g). The imaging region is set to be around the red-dashed circle in Fig. 3(b). Figure 3(d) shows an image with wavefront shaping; Fig. 3(e) shows an image acquired using uncontrolled illumination; Fig. 3(f) is an image acquired using the spatial compounding method. The images of Figs. 3(d)-3(f) were all acquired from the same incident beam power of $0.55 \mathrm{~mW}$. The images obtained using the uncontrolled illumination and spatial compounding methods show similar penetration depths and signal levels, but we can see that these two conventional imaging methods have large shortcomings compared to the wavefront shaping method regarding penetration depth and signal-to-noise ratio (SNR). For further comparison, the image in Fig. 3(g) was acquired using uncontrolled illumination but with a doubled incident power of $1.1 \mathrm{~mW}$. The wavefront shaping image shows a comparable level of SNR and even slightly more features below layer II compared with the sample subject to doubled power illumination. To quantitatively compare the signal levels for the four cases, we plotted the averaged signal profiles over $25 \mathrm{~A}$-scans versus depth. We can see that wavefront shaping yields the largest signal over the depth ranges. The signal levels exponentially decay as the depth increases, so layer I is the only accessible imaging area even in wavefront shaping.

After demonstrating the enhanced penetration depth with wavefront shaping in the original sample state, we next applied the OCA to the same region and acquired images with the same procedures as used above. After adjusting only the $z$-position of the sample stage, we applied $300 \mu \mathrm{L}$ of $70 \%$ glycerol solution to the imaging region and restored the sample to the original position. We waited for $10 \mathrm{~min}$ after the application of the OCA to allow OCA to diffuse into the sample. A wavefront shaping image acquired after the OCA is shown in Fig. 3(i). The third layer became visible; it had not been possible to visualize this layer using any of the previous methods. Also, the signal level decreased in the phantom layer, which implies that the OCA successfully penetrated into the tissue phantom and made the sample more transparent. This effect can also be observed in the uncontrolled beam image and the spatial compounding image, as shown in Figs. 3(j) and 3(k), respectively. Since the scattering of the phantom sample is significantly reduced, no meaningful signal having structural information is observed in layer I. Figure 3(1) shows an image acquired using double powered uncontrolled illumination. 
(a)
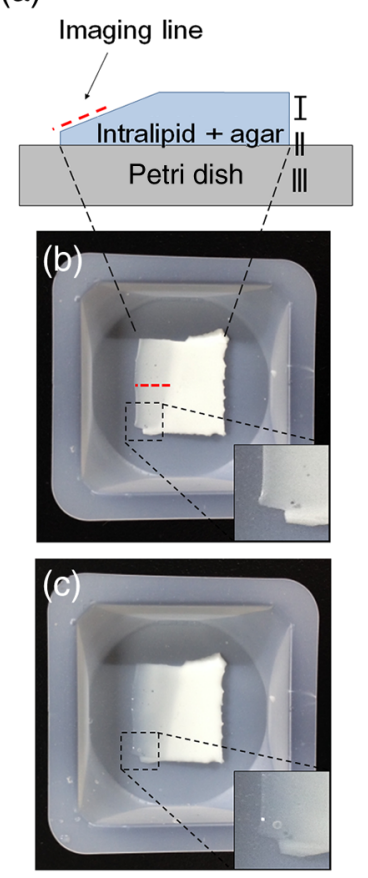
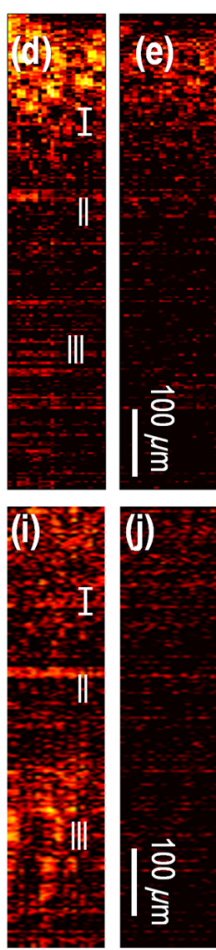
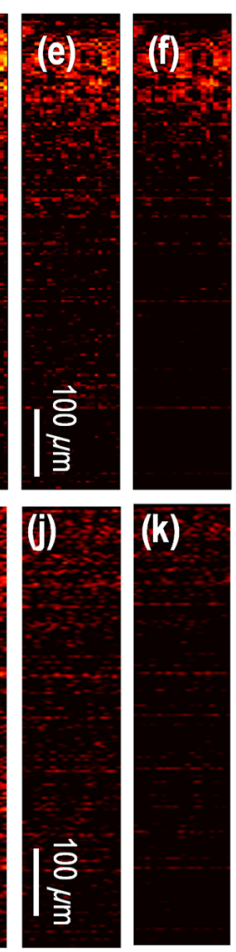

(k)
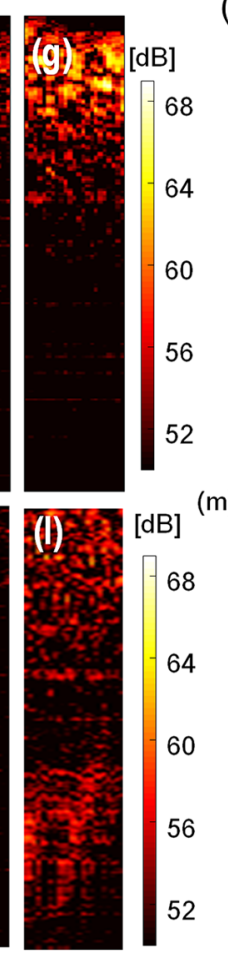

(h)
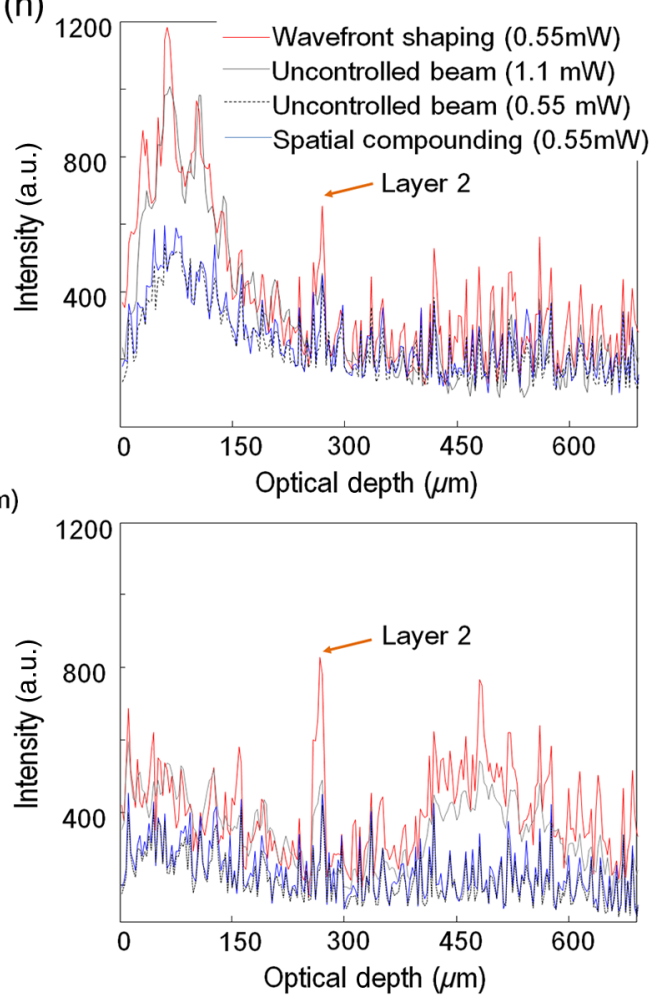

Fig. 3 (a) Structure of a tissue phantom. Photograph of the tissue phantom (b) before applying the OCA and (c) after applying the OCA. (inset) Magnified views of the edges. (d)-(h) Images acquired before the OCA. (i)-(m) Images acquired after the OCA. (d)-(i) Optimized image obtained by wavefront control with input power of $0.55 \mathrm{~mW}$. (e)-(j) Images acquired for an uncontrolled beam of $0.55 \mathrm{~mW}$. (f)-(k) Images obtained by spatial compounding method. (g)-(I) Images acquired for uncontrolled beams of $1.1 \mathrm{~mW}$. (h)-(m) The averaged A-scan profiles along 25 A-scans were plotted for each method.

The average profiles over $25 \mathrm{~A}$-scans are plotted in Fig. 3(m). Compared to the signal profiles obtained before the OCA, the signal level has decreased in layer I and the signals from the Petri dish in layer III have increased against the background signal level. Without the OCA, the maximum penetration depth for wavefront shaping is $<300 \mu \mathrm{m}$, as can be seen in Fig. 3(h). With the OCA and wavefront shaping, structures deeper than $600 \mu \mathrm{m}$ from the surface became accessible, as can be seen in Fig. 3(m), which shows a more than two-fold enhancement in the penetration depth.

\section{Ex Vivo Mouse Ear}

To test the suitability of our technique for real biological samples, we next applied the OCA and wavefront shaping to an ex vivo mouse ear. In the tissue phantom study, the optical inhomogeneity of the Petri dish was not affected by the application of the OCA, and these preserved scattering contrasts were easily imaged using WS-OCT. For real biological samples, however, all imaging regions are optically cleared by the OCA. Therefore, it is important to confirm the proposed technique in real biological tissues.

\subsection{Sample Preparation}

After anesthetization with an intraperitoneal injection of tiletamine-zolazepam and xylazine mixture $(30: 10 \mathrm{mg} / \mathrm{kg}$ body weight), a mouse ear was dissected from a specific pathogenfree C57BL/6J mouse (Jackson Laboratory). All experimental procedures were approved by the Institutional Animal Care and Use Committee (IACUC) of the Korea Advanced Institute of Science and Technology (KAIST). After the dissection, the ear was immersed in $70 \%$ glycerol solution for $1 \mathrm{~h}$. A photograph of the whole mouse ear is shown in Fig. 4(a); the representative histology of the subregion is shown in Fig. 4(b). To compare the histology and the OCT images, subsequent OCT signals are plotted according to physical depth while assuming a refractive index of 1.4 for the mouse skin. ${ }^{6}$ For example, a $140-\mu \mathrm{m}$ optical depth in OCT signal is converted to a $100-\mu \mathrm{m}$ physical depth. This scale factor can vary upon the application of OCAs. Since the refractive index of $70 \%$ glycerol is 1.463 , the change in the scale factor is $<5 \%$ even under the assumption that the sample medium is completely replaced with glycerol. This change corresponds to the $12-\mu \mathrm{m}$ for a $300-\mu \mathrm{m}$-thickness sample. Thus, we assume that there is no significant effect of the OCA on the comparison between the histology and the OCT images.

\subsection{Experimental Results and Discussion}

Three different locations on the mouse ear were imaged before OCA, and three other locations were imaged after the application of OCA. The OCT images before OCA are first presented in Figs. 4(c)-4(h). For clear comparisons, we show here only the wavefront shaping images and the uncontrolled beam images for the same incident power of $0.55 \mathrm{~mW}$. The wavefront shaping images [Figs. 4(c), 4(e), and 4(g)] show superficial layers of the ear tissue, whereas image signals cannot be clearly seen with the uncontrolled beam illumination, as shown in Figs. 4(d), 4(f), and 


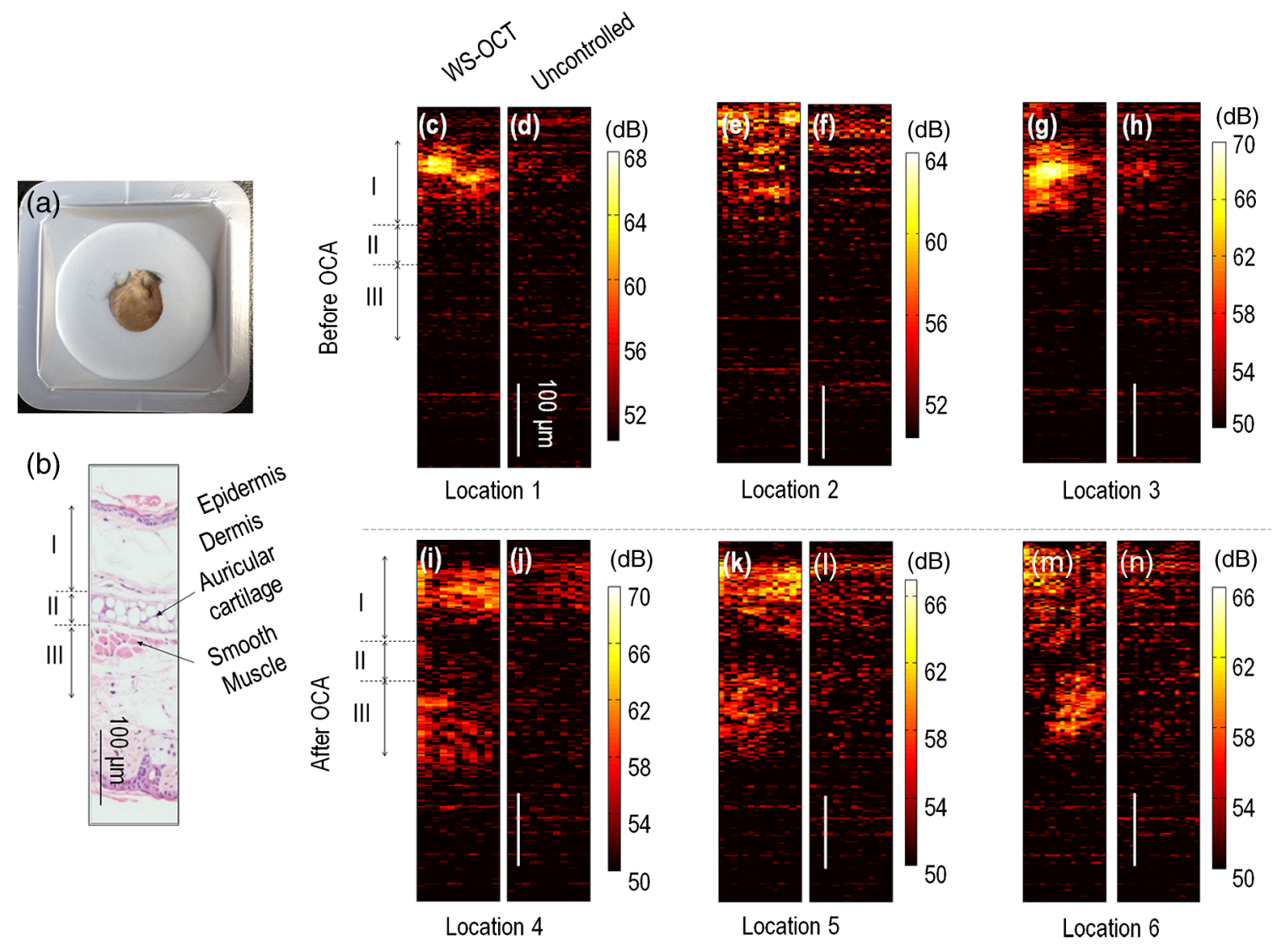

Fig. 4 (a) Dissected mouse ear; (b) histology of the ear; [(c)-(h) not shown] images acquired before the OCA; [(i)-(n) not shown] images acquired after the OCA; [(c), (e), (g), (i), (k), and (m) not shown)] image optimized by wavefront control with the input power of $0.55 \mathrm{~mW}$ at locations 1 to 6 ; [(d), (f), (h), (j), (l), and (n) not shown] images acquired for uncontrolled beam of $0.55 \mathrm{~mW}$ at locations 1 to 6.

4(h). Although wavefront shaping provides enhanced penetration depth and SNR over those characteristics of uncontrolled beam illumination, only a single layer is visible, and the structural information of the ear is hardly attainable even in the wavefront shaping case.

To achieve more enhancement of the penetration depth using OCA, we immersed the ear in the $70 \%$ glycerol solution and acquired OCT images, which are shown in Figs. 4(i)-4(n). Since the whole ear was taken off of the sample stage and immersed in the OCA solution, locating the original imaging positions was impossible, so we assumed that the penetration depth was constant for nearby imaging areas. As can be clearly seen in Figs. 4(i), 4(k), and 4(m), the wavefront shaping technique provides remarkable enhancements: the double layered structures can be visualized for every location. For the uncontrolled input beam cases [Figs. 4(j), 4(1), and 4(n)], the signal strengths are slightly better than those acquired before the OCA, but still they provide blurred images of the tissue structures.

Figure 4(b) shows a representative histology image taken from the middle ear of a mouse that may reflect the multilayer structure found in Figs. 4(i), 4(k), and 4(m). It should be noted that the histology and OCT images were acquired from different ears. However, we assumed that the general structure is about the same in ears of the same species. In particular, for the case shown in Fig. 4(i), we speculate that the three layers (I, II, and III) match the epidermis, auricular cartilage, and smooth muscle, as found in Fig. 4(b).
To compare these data quantitatively, we plotted the averaged depth profiles over 25 A-scans in Figs. 5(a)-5(f) for each location, as can be seen in Fig. 4. The same trends as those found in the cases of the tissue phantom study are observed: (1) wavefront shaping gives the highest signal level in all locations and (2) the uncontrolled beam illumination with $0.55 \mathrm{~mW}$ and the spatial compounding method with $0.55 \mathrm{~mW}$ give the smallest SNR. When the wavefront shaping cases are considered exclusively, imaging depths are limited up to $\sim 200 \mu \mathrm{m}$ before the OCA and the penetration depth reaches $300 \mu \mathrm{m}$ after applying the OCA.

The results above clearly show the collaborative effects of wavefront shaping and OCA in OCT. Using scattering phantom samples and in vitro biological tissue samples, we have demonstrated that both the penetration depth and the SNR can be significantly improved, compared to the case of using conventional OCT without OCA treatment. Importantly, the simultaneous application of wavefront shaping and OCA results in collaborative effects for deep-tissue optical imaging.

For future studies, the optimal conditions of the OCA treatment and wavefront shaping should be further investigated. For example, various OCAs are available for different tissues types and imaging conditions. ${ }^{19,55,56}$ The optimal selection of OCAs will provide more enhanced penetration depths. The wavefront shaping technique also has much room for improvement. In our previous results, ${ }^{37}$ the depth enhancement factor increases as the scattering of the tissue phantom is reduced. This trend shows a positive sign that further optimization of collaborative effect can 
(a)

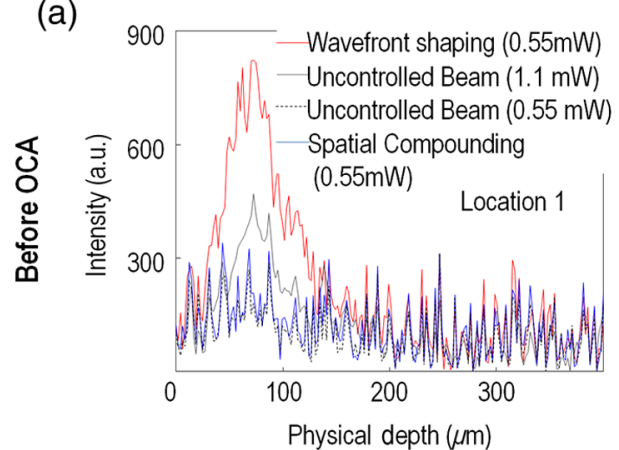

(d)

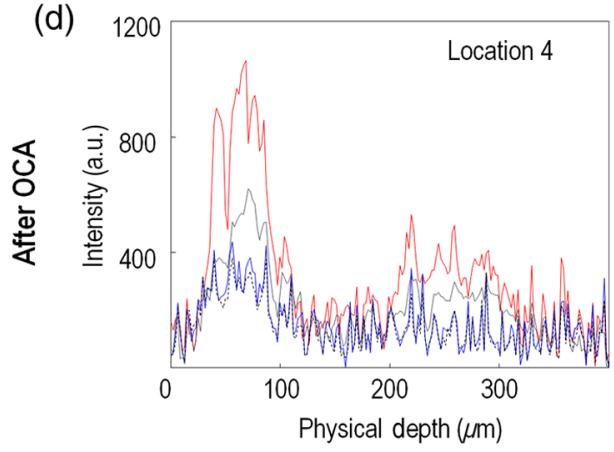

(b)

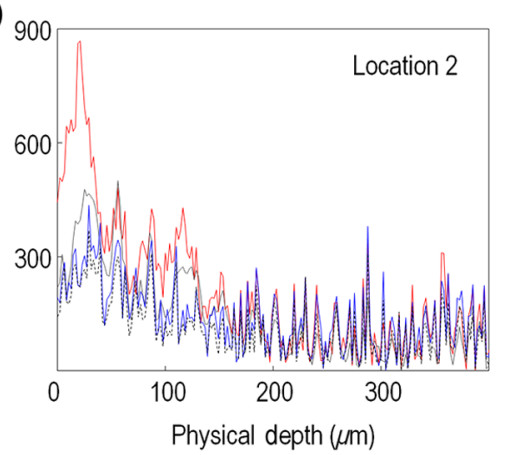

(e)

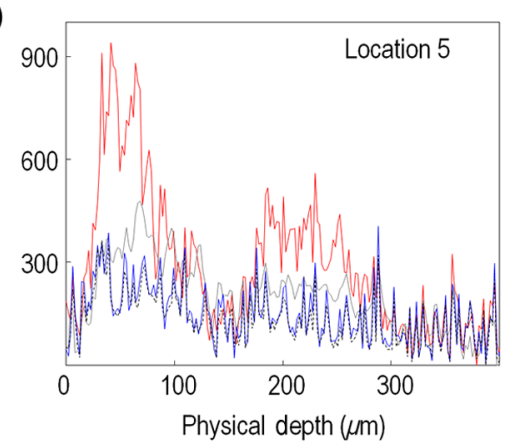

(c)

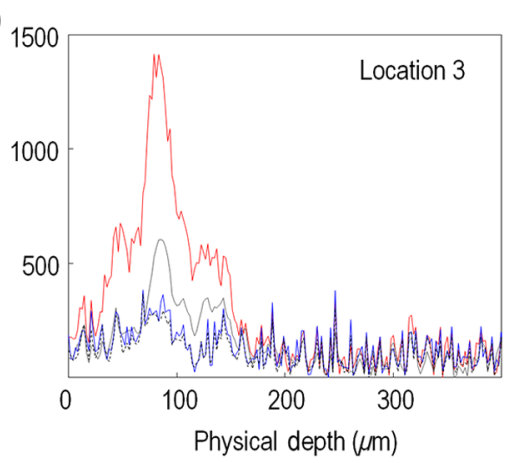

(f)

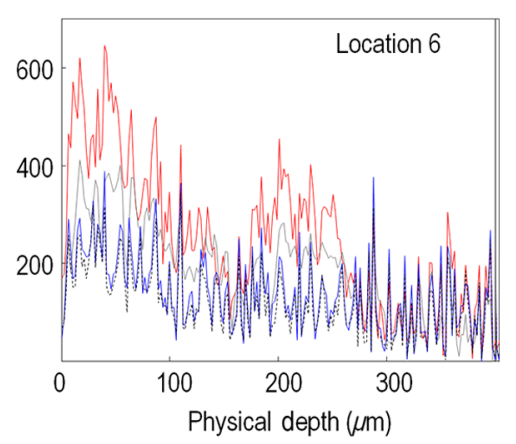

Fig. 5 The averaged A-scan profiles along 25 A-scans for each method (a)-(c) before the OCA and (d)-(f) after the OCA at locations 1 to 6.

lead to the synergetic effect of the both method, where the depth enhancement is greater than the sum of individual effects. Although we focused on the reduced scattering coefficient of the sample, the effects of the variation on the anisotropy factor and the scattering coefficient are still an open question. Proper setting of the wavefront parameter according to the sample conditions is expected to bring about higher enhancements of the penetration depth.

In the current approach, there is partial loss of sample information due to OCAs, as seen in the optical clearing of the phantom layer in the tissue phantom study. Furthermore, there should be balanced applications of OCAs. The wavefront shaping utilizes backscattered signals as a feedback signal. Thus, the reduction of scattering can prevent efficient wavefront shaping. For example, the complete clearing of OCAs makes the present approach inapplicable. The minimum amount of scattering for collaborative effects should be further studied.

The ultimate goal of deep-tissue imaging is to achieve penetration depth enhancement in vivo. ${ }^{23,57,58}$ We anticipate that in vivo application can be realized in the near future because OCA and WS-OCT have worked successfully in in vivo environments. However, many experimental difficulties have to be overcome. First, the delivery of the OCA into living tissues is not straightforward and usually requires special treatments. ${ }^{21,59}$ Second, the image acquisition speed with wavefront shaping must be further optimized. Recently, we demonstrated that the wavefront shaping OCT works for static biological tissues in vivo. ${ }^{39}$ However, the 15-s optimization time for a single A-scan is too slow to image moving tissues. To be applicable for in vivo imaging, the wavefront shaping should as fast as the aberration correction in sensorless adaptive optics OCT. ${ }^{60,61}$ To enhance the optimization speed, various approaches can be applied, such as the computation time reduction using graphics processing unit (GPU), implementing the wavefront shaping in swept-source OCT platform, or designing efficient optimization algorithm based on preacquisition plain OCT data. If these problems are adequately addressed, wavefront shaping and OCAs will provide a promising method for in vivo deep-tissue imaging.

\section{Conclusion}

Here, we have demonstrated penetration depth enhancement in WS-OCT by simultaneously using wavefront shaping and OCAs. With the application of $70 \%$ glycerol solution as an OCA and the wavefront shaping technique via a DMD, we visualized multilayered structures of a tissue phantom and ex vivo mouse ear with unprecedented penetration depth enhancement in comparison to that possible with conventional OCT imaging. For the image optimized with wavefront shaping, the application of OCA provided an additional enhancement factor of 1.5 to 2 for the penetration depth.

\section{Acknowledgments}

This work was supported by KAIST, the National Research Foundation of Korea (Nos. 2015R1A3A2066550, 2014K1A3A1A09063027, and 2014M3C1A3052537), and Innopolis Foundation (No. A2015DD126). V. V. T. was supported by Russian Presidential under Grant No. NSh-7898 .2016 .2 and the Russian Government under Grant No. 14.750 .31.0004.

\section{References}

1. D. Huang et al., "Optical coherence tomography," Science 254(5035), 1178-1181 (1991).

2. A. F. Fercher et al., "In-vivo optical coherence tomography," Am. J. Ophthalmol. 116(1), 113-114 (1993).

3. E. A. Swanson et al., "In-vivo retinal imaging by optical coherence tomography," Opt. Lett. 18(21), 1864-1866 (1993). 
4. W. Drexler and J. G. Fujimoto, Eds., Optical Coherence Tomography: Technology and Applications, 2nd ed., Springer Science \& Business Media, Switzerland (2015).

5. J. Yao and L. V. Wang, "Photoacoustic microscopy," Laser Photonics Rev. 7(5), 758-778 (2013).

6. D. A. Boas, C. Pitris, and N. Ramanujam, Handbook of Biomedical Optics, CRC Press, Boca Raton (2011).

7. R. K. K. Wang, "Signal degradation by coherence tomography multiple scattering in optical of dense tissue: a Monte Carlo study towards optical clearing of biotissues," Phys. Med. Biol. 47(13), 2281-2299 (2002).

8. D. Zhu et al., "Recent progress in tissue optical clearing," Laser Photonics Rev. 7(5), 732-757 (2013).

9. V. V. Tuchin, "Optical clearing of tissues and blood using the immersion method," J. Phys. D Appl. Phys. 38(15), 2497-2518 (2005).

10. V. V. Tuchin et al., "Light propagation in tissues with controlled optical properties," J. Biomed. Opt. 2(4), 401-417 (1997).

11. R. LaComb et al., "Quantitative second harmonic generation imaging and modeling of the optical clearing mechanism in striated muscle and tendon," J. Biomed. Opt. 13(2), 021109 (2008).

12. X. Wen et al., "Controling the scattering of Intralipid by using optical clearing agents," Phys. Med. Biol. 54(22), 6917-6930 (2009).

13. B. Choi et al., "Determination of chemical agent optical clearing potential using in vitro human skin," Laser Surg. Med. 36(2), 72-75 (2005).

14. Z. Z. Mao et al., "Influence of alcohols on the optical clearing effect of skin in vitro," J. Biomed. Opt. 13(2), 021104 (2008).

15. A. T. Yeh et al., "Reversible dissociation of collagen in tissues," J. Invest. Dermatol. 121(6), 1332-1335 (2003).

16. J. Hirshburg et al., "Correlation between collagen solubility and skin optical clearing using sugars," Laser Surg. Med. 39(2), 140-144 (2007).

17. W. Feng et al., "Skin optical clearing potential of disaccharides," J. Biomed. Opt. 21(8), 081207 (2016).

18. C. G. Rylander et al., "Dehydration mechanism of optical clearing in tissue," J. Biomed. Opt. 11(4), 041117 (2006).

19. E. A. Genina et al., "Optical clearing of human skin: comparative study of permeability and dehydration of intact and photothermally perforated skin," J. Biomed. Opt. 13(2), 021102 (2008).

20. L. Oliveira et al., "Optical characterization and composition of abdominal wall muscle from rat," Opt. Lasers Eng. 47(6), 667-672 (2009).

21. I. V. Larina et al., "Enhanced OCT imaging of embryonic tissue with optical clearing," Proc. SPIE 7168, 71682C (2009).

22. S. G. Proskurin and I. V. Meglinski, "Optical coherence tomography imaging depth enhancement by superficial skin optical clearing," Laser Phys. Lett. 4(11), 824-826 (2007).

23. X. Wen et al., "Enhanced optical clearing of skin in vivo and optical coherence tomography in-depth imaging," J. Biomed. Opt. 17(6), 066022 (2012).

24. O. Zhernovaya, V. V. Tuchin, and M. J. Leahy, "Blood optical clearing studied by optical coherence tomography," J. Biomed. Opt. 18(2), 026014 (2013).

25. M. G. Ghosn, V. V. Tuchin, and K. V. Larin, "Depth-resolved monitoring of glucose diffusion in tissues by using optical coherence tomography," Opt. Lett. 31(15), 2314-2316 (2006).

26. M. Kinnunen et al., "In vitro studies toward noninvasive glucose monitoring with optical coherence tomography," Appl. Opt. 45(10), 2251-2260 (2006).

27. M. G. Ghosn et al., "Monitoring of glucose permeability in monkey skin in vivo using optical coherence tomography," J. Biophotonics 3(1-2), 25-33 (2010).

28. M. G. Ghosn et al., "Differential permeability rate and percent clearing of glucose in different regions in rabbit sclera," J. Biomed. Opt. 13(2), 021110 (2008).

29. K. V. Larin et al., "Optical clearing for OCT image enhancement and indepth monitoring of molecular diffusion," IEEE J. Sel. Top. Quantum Electron. 18(3), 1244-1259 (2012).

30. X. Guo et al., "In vivo quantification of propylene glycol, glucose and glycerol diffusion in human skin with optical coherence tomography," Laser Phys. 20(9), 1849-1855 (2010).

31. B. Hermann et al., "Adaptive-optics ultrahigh-resolution optical coherence tomography," Opt. Lett. 29(18), 2142-2144 (2004).

32. R. Zawadzki et al., "Adaptive-optics optical coherence tomography for high-resolution and high-speed 3D retinal in vivo imaging," Opt. Express 13(21), 8532-8546 (2005).
33. A. P. Mosk et al., "Controlling waves in space and time for imaging and focusing in complex media," Nat. Photonics 6(5), 283-292 (2012).

34. H. Yu et al., "Recent advances in wavefront shaping techniques for biomedical applications," Curr. Appl. Phys. 15(5), 632-641 (2015).

35. I. M. Vellekoop and A. Mosk, "Focusing coherent light through opaque strongly scattering media," Opt. Lett. 32(16), 2309-2311 (2007).

36. R. Fiolka, K. Si, and M. Cui, "Complex wavefront corrections for deep tissue focusing using low coherence backscattered light," Opt. Express 20(15), 16532-16543 (2012).

37. H. Yu et al., "Depth-enhanced 2-D optical coherence tomography using complex wavefront shaping," Opt. Express 22(7), 7514-7523 (2014).

38. J. Jang et al., "Complex wavefront shaping for optimal depth-selective focusing in optical coherence tomography," Opt. Express 21(3), 2890 $2902(2013)$

39. H. Yu et al., "In vivo deep tissue imaging using wavefront shaping optical coherence tomography," J. Biomed. Opt. 21(10), 101406 (2016).

40. Y. Choi et al., "Measurement of the time-resolved reflection matrix for enhancing light energy delivery into a scattering medium," Phys. Rev. Lett. 111(24), 243901 (2013).

41. S. Kang et al., "Imaging deep within a scattering medium using collective accumulation of single-scattered waves," Nat. Photonics 9(4), 253258 (2015).

42. A. Badon et al., "Smart optical coherence tomography for ultra-deep imaging through highly scattering media," arXiv preprint arXiv: 1510.08613 (2015).

43. N. Ji, D. E. Milkie, and E. Betzig, "Adaptive optics via pupil segmentation for high-resolution imaging in biological tissues," Nat. Methods 7(2), 141-147 (2010).

44. J.-H. Park, W. Sun, and M. Cui, "High-resolution in vivo imaging of mouse brain through the intact skull," Proc. Natl. Acad. Sci. 112(30), 9236-9241 (2015).

45. A. F. Fercher et al., "Optical coherence tomography-principles and applications," Rep. Prog. Phys. 66(2), 239-303 (2003).

46. A. E. Desjardins et al., "Angle-resolved optical coherence tomography with sequential angular selectivity for speckle reduction," Opt. Express 15(10), 6200-6209 (2007).

47. N. Iftimia, B. E. Bouma, and G. J. Tearney, "Speckle reduction in optical coherence tomography by 'path length encoded' angular compounding," J. Biomed. Opt. 8(2), 260-263 (2003).

48. M. Pircher et al., "Speckle reduction in optical coherence tomography by frequency compounding," J. Biomed. Opt. 8(3), 565-569 (2003).

49. M. Szkulmowski et al., "Efficient reduction of speckle noise in optical coherence tomography," Opt. Express 20(2), 1337-1359 (2012).

50. J. Y. Jiang et al., "Quantitative control of optical clearing effects studied with tissue-like phantom," J. Innov. Opt. Heal. Sci. 3(3), 195-202 (2010).

51. B. W. Pogue and M. S. Patterson, "Review of tissue simulating phantoms for optical spectroscopy, imaging and dosimetry," J. Biomed. Opt. 11(4), 041102 (2006)

52. S. A. Prahl, M. J. C. Vangemert, and A. J. Welch, "Determining the optical-properties of turbid media by using the adding-doubling method," Appl. Opt. 32(4), 559-568 (1993).

53. H. J. Vanstaveren et al., "Light-scattering in intralipid-10-percent in the wavelength range of 400-1100 Nm," Appl. Opt. 30(31), 4507-4514 (1991).

54. H. F. Ding et al., "Determination of refractive indices of porcine skin tissues and Intralipid at eight wavelengths between 325 and $1557 \mathrm{~nm}$," J. Opt. Soc. Am. A 22(6), 1151-1157 (2005).

55. B. Lei et al., "Monitoring of permeability of different analytes in human normal and cancerous bladder tissues in vitro using optical coherence tomography," Quantum Electronics 44(12), 1165-1169 (2014).

56. Z. Zhu et al., "Investigation of the permeability and optical clearing ability of different analytes in human normal and cancerous breast tissues by spectral domain OCT," J. Biophotonics 5(7), 536-543 (2012).

57. E. A. Genina et al., "Optical coherence tomography monitoring of enhanced skin optical clearing in rats in vivo," J. Biomed. Opt. 19(2), 021109 (2014).

58. M. Kuck et al., "In vivo enhancement of imaging depth for optical coherence tomography by eudermic agents on ridged and meshed human skin," Laser Phys. Lett. 11(3), 035602 (2014).

59. K. Chung et al., "Structural and molecular interrogation of intact biological systems," Nature 497(7449), 332-337 (2013). 
60. Y. Jian et al., "Wavefront sensorless adaptive optics optical coherence tomography for in vivo retinal imaging in mice," Biomed. Opt. Express 5(2), 547-559 (2014).

61. H. Hofer et al., "Wavefront sensorless adaptive optics ophthalmoscopy in the human eye," Opt. Express 19(15), 14160-14171 (2011).

Hyeonseung Yu received his BS degree in physics and mathematical science from Korea Advanced Institute of Science and Technology (KAIST). He is currently a PhD student in the Department of Physics at KAIST.

Peter Lee received his BSE and MSE degrees in bioengineering from the University of Pennsylvania. He is currently a PhD student in the Department of Bio and Brain Engineering at KAIST.

YoungJu Jo is currently a BS student in the Department of Physics at KAIST.

KyeoReh Lee received his BS degree in physics from KAIST. He is currently a PhD student in the Department of Physics at KAIST.

Valery V. Tuchin is a professor and chairman of optics and biophotonics at Saratov National Research State University and several other universities and institutions. His research interests include tissue optics, laser medicine, tissue optical clearing, and nanobiophotonics. He is a fellow of SPIE and OSA, has been awarded Honored Science Worker of the Russia, SPIE Educator Award, FiDiPro (Finland), Chime Bell Prize of Hubei Province (China), and Joseph W. Goodman Book Writing Award (OSA/SPIE).

Yong Jeong received his MD degree in 1991 and a PhD in neurophysiology in 1997 from Yonsei University. He is an associate professor at the Department of Bio and Brain Engineering at KAIST and deputy director of $\mathrm{KI}$ for Optical Science and Engineering. His research fields are cognitive neuroscience, clinical neurology (degenerative disease, vascular disease), functional neuroimaging, and bioengineering (biosignals). He is also a neurologist and is affiliated with Samsung Medical Center.

YongKeun Park received his $\mathrm{PhD}$ in medical physics and medical engineering from Harvard-MIT Health Science and Technology in 2010 and joined KAIST. He is an associate professor in the Department of Physics at KAIST. He is an associate editor of Experimental Biology and Medicine, and editorial board member of Scientific Reports, Optics Express and Journal of Optical Society of Korea. 